\title{
Research and application of grouting material in assembled concrete structure
}

\author{
Pei-Nan Zhang ${ }^{1}$, Ming $\mathrm{Li}^{1}$, and Yan-Ting Hao ${ }^{1}$ \\ ${ }^{1}$ Shenyang Jianzhu University, Shenyang, 110168, China. \\ animozhang@foxmail.com, bdingxianxing@126.com, chao_yanting@163.com
}

\begin{abstract}
Keywords: grouting material; assembled concrete; connection; research progress
Abstract: The assembled concrete structure has the advantages of energy saving, environmental protection and high construction efficiency. It becomes one of the important directions in the development of civil engineering. Grouting material is an important material of the prefabricated components connection at present. So, it's introduced the typical form of grouting material in the application of assembled concrete structure this paper, including beam-to-column connection, column-to-column connection, wall-to-wall connection. This paper also introduces the stages of the developing course and classification of grouting material. The former includes the original clay slurry grouting stage, the early cement grouting stage, medium-term chemical grouting stage and modern grouting stage, the later includes solid particles grouting material, chemical grouting material and fine mineral grouting material. Based on that, it's pointed out the problems existing in the research and the research direction of the grouting material in the assembled concrete structure. Finally, it's discussed the problems needed to be studied in the future.
\end{abstract}

\section{Introduction}

Assembled concrete structure, which has the advantages of energy conservation, emission reduction and high construction efficiency, has been highly valued and developed in the United States, Japan, China and other countries. Especially in recent years, the development of assembly type construction in China is more rapid. In the Assembled concrete structure, the connection between prefabricated components is implementation difficulties and key. The connection of steel is an important factor in determining the quality of precast element connections. At present, with the construction of highway, bridge, dam and other large engineering projects, and advantages of the practical, convenient grouting material has been widely used and developed. However, because of the different advantages and disadvantages of grouting materials, the application of grouting materials in the assembled concrete structure is less. So we need to find a more reasonable form of grouting material from the material point of view. For this purpose, this paper will review the application of grouting material in the assembled concrete structure and its research progress, and provide reference for the future research in this field.

\section{Application method of grouting material in assembled concrete structure}

In the assembled concrete structure, grouting material is applied to the important part of the structural member, and there are many kinds of connection forms. The following will make a summary of the application of the grouting material in the assembled concrete structure, and provide reference for the research of the material engineer in this field.

\subsection{Beam-to-column connection}

In the assembled concrete structure, the typical node form of beam-to-column connections is as shown in Fig1. First make the sleeve is sheathed on the steel bar of the beam, then adjust beam and column to a predetermined position, and make steel bar which was embedded in the column be aligned with the beam, and then moves the sleeve from the beam to the steel bar. Finally grouting in the sleeve, complete the connection of steel bar. Grout concrete in the beam-column connection part, complete the connection of beam-to-column. 


\subsection{Column-to-column connection}

In the assembled concrete structure, the typical node form of column-to-column connections is as shown in Fig2, In the figure,one end of the reinforcement of precast column extends into the sleeve, the other end extends out of the precast column. When they are connected, the reinforcement of the precast column extends to the sleeve of the other precast column. Then grouting in the sleeve,complete the connection of column-to-column.

\subsection{Wall-to-wall connection}

In the assembled concrete structure, there are many reinforcementin shear wall, Generally we seeing vertical reinforcements of the shear wall as a reinforcement. One end of the reinforcements of shear wall connected to the sleeve into the wall, the other end extends out of the shear wall. Shown as Fig3. When they are connected, the reinforcement of the shear wall extends to the sleeve of the other shear wall. Then the grout material is grouted in the sleeve and the vertical connection of shear wall is completed.

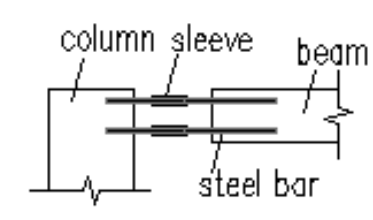

Fig1.Beam-to-column connection

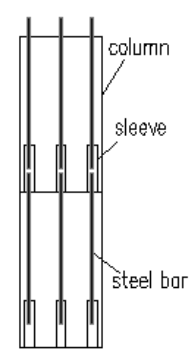

Fig2. Column-to-column connection

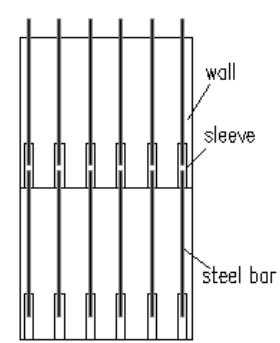

Fig3. Wall-to-wall connection

\section{The developing course of grouting material}

Grouting material is roughly experienced four stages: the original clay slurry grouting stage (18021857), the early cement grouting stage (1858-1858), medium-term chemical grouting stage (1920-1920) and modern grouting stage (after 1969) [1].

In the original clay slurry grouting stage, grouting technology is in the original embryonic stage, the injection method is original, and the slurry is mainly consist of clay, volcanic ash, quicklime and other simple materials.

In the early cement grouting stage, People began using cement as grouting material, especially Germany and Belgium developed the high pressure grouting pump , which improved the hybrid way of grouting materials and grouting technology, formed the foundation of modern grouting method.

In the Mid-term chemical grouting stage, the sodium silicate injection method is mainly appeared. Because of the price is cheap and non-toxic, sodium silicate had a rapid development. There are inorganic, organic and all kinds of new materials as chemical grouting materials. However, due to chemical grout material often caused certain environmental pollution or poisoning, the use of chemical grouting material also has certain restrictions in the late 70s of last century.

In the Modern grouting stage, due to chemical grouting material has poison, and sodium silicate grouting can't meet the needs of large project in consolidation strength and durability aspects. As a result, people began to turn the target to the development and utilization of Portland cement, then Japan, the United States and other countries have developed the superfine cement, Wet grinding cement grouting technology, and claimed its application range is generally equivalent to chemical grouting material[2]. 


\section{The research progress of grouting material}

With the rapid development of domestic and foreign construction, grouting material has been widely developed and utilized. From lime and clay, cement, to a variety of chemical grouting material, super fine cement, etc. It can be roughly divided into solid particles grouting material, chemical grouting material and fine mineral grouting material.

\section{1 solid particles grouting material}

Solid particles grouting material consists of solid particles and water. It is convenient, cheap, simple, and has a good ability of seepage-proofing and consolidation, but the gap width of the grouting can be limited by the size of solid particles. Solid particles grouting material can be roughly divided into clay grout, cement grout, cement-clay grout and cement-fly ash grout.

Clay grout is the earliest grouting material. The clay particles have many advantages such as low water permeability, excellent stability and the cheap price, but the set strength and bond force are very low, and the ability of anti-permeability pressure is weak. Cement grout is the most widely used grouting material. They have many advantages such as high cementing power and high set strength and it is also quite convenient. Used for reinforcement and seepage-proofing of rock, foundation or structure, the joint treatment of dams, the duct grouting of post tensioned prestressed concrete, and the production of grouting concrete. Cement clay grout can choose different cement clay mixture ratio in different environment, it synthesizes the strength of cement grout and the high strength, good stability and cheap price of clay grout. Cement-fly ash grout is increasingly widely used in the grouting engineering because of fine particles of fly ash.

There are a lot of research on grouting material of cement grout, typically include: Yuan $\mathrm{Wu}$ studied how the cement-based grouting type, water addition, the content of pisolite influences the mechanical properties of cement grouting material through 201 cube specimens and 89 prism specimens of mechanical properties test[3]. Based on the test data, the formula of size effect, strength transformation and age strength of cement based grouting material are given. Shi-Bao Zhang determined the mix radio meets the engineering requirements and easy construction of cement clay composite grouting material by experiment[4]. Li-Qiang Huo used fly ash, slag, such as ordinary Portland cement as the main material, mixed with homemade admixture, made high-performance green grout[5].

There are some problems in the solid particles grouting material, such as the low water retention of cement based grouting material, and the limited grouting range, which can only be poured into the pores and cracks with large diameter. In addition, the domestic superfine cement variety and performance are single. And the need to cost a lot of land resources, long grout setting time is also limited its application.

\subsection{Chemical grouting material}

A liquid that is prepared by a chemical agent, apply to the more subtle gap and it can adjust the setting time according to need. Chemical grouting material is divided into inorganic and organic chemical grout.

Inorganic grouting materials use sodium silicate as the main material of chemical grout. There are two fluid methods, i.e. double-fluid method and Single-fluid method. Double-fluid method is used for the consolidation and seepage-proofing of the sandy soil with large permeability coefficient, and Single-fluid method is used for the reinforcement of the loess or loess like soil.

Organic grouting materials using various polymer materials as the main raw materials, such as epoxy resin, methyl methacrylate and acrylamide etc. Epoxy resin grouting material has strong bonding strength, low shrinkage and good stability. It is the main reinforcement material of the structural concrete. Methyl methacrylate grouting material has a lower viscosity, strong permeability, It has high post polymerization strength and high bond strength, can be poured into the tiny fracture, and commonly used in concrete dam, tubing, dock and the basis of fill and plugging. The viscosity of acrylamide grouting material is very low, It can be poured into the gap 
where the cement grout can't reach, which is suitable for the seepage-proofing project.

The study of chemical grouting material, developed along with the development of chemical drugs. Such as Yong-Zhen Wang made the low modulus and high elastic epoxy grouting material by using eighteen amine and ethylene glycol ether as raw materials[6]. Xiang-Yang Liu used formamide as curing agent prepared a kind of chemical grouting material with high temperature resistance, good groutability, high solidified compression strength[7]. And it is harmless to the human body and the environment, which is used for gas under-balanced drilling in complex stratum. Wen Hestudied the solution type sodium silicate chemical grouting material to solve the grouting difficult problem in deep coal mine in the bedrock section and porosity aquifer stratum[8]. Experimental results show that using ethylene glycol diacetate as gelling agent of sodium silicate can get high consolidating strength, short gelation time, and low stiffness new sodium silicate grout.

The problem of chemical grouting material is that its formula is complex, the performance is single, contain more toxic chemicals, not only pollute the environment, but also whose price is more expensive.

\subsection{Fine mineral grouting material}

Fine mineral grouting material is a new type of grouting material, formed by the combination of different natural minerals, artificial minerals and special functional materials and achieved a breakthrough in the grout performance, consolidation performance, long-term durability and other aspects of the key performance. Some fine mineral has been close to or better than the performance of the chemical grouting material in grout properties, grout stability, grout viscosity, injection ability, adjusted gelation time, the consolidation strength and other important properties. Such as Wuhan Polytechnics University used pulverized slag completely replace the cement. They excited industrial waste residue by sodium silicate and made different gelation time, high compression strength and 100\% stone rate new double-fluid grouting material, and applied to the construction of Wuhan Yangtze River tunnel project successfully. So, the method of the grouting material prepared by using the industrial waste slag, such as fly ash, slag, steel slag and so on is not only conducive to a large number of industrial waste residue waste become to treasure, reduce environmental pollution, reduce the cost of grouting material.

New fly ash, slag, steel slag and other solid waste production is acceptable to meet engineering requirements in the macroscopic properties, But it's adaptation in early stage and durability in later period haven't been verified by experiment yet.

\section{Conclusion and prospect}

The above statements summarized the situation of application and research about grouting beam in assembled concrete structure, from which we know that:

1 .Different grouting materials have different scope of application and parochialism. Their mobility, gelation time, stability, toxicity, compressive, reinforcement, extension alrigidity, anti-permeability, corrosion resistant ability, aging resistance and other performances are very different. Therefore, it is necessary to carry out comprehensive comparison and experimental research about performance of various kinds of grouting material.

2. Nowadays, traditional cement-based grouting material is still the main grouting material in assembled concrete structure. Because it still exists malpractices, we need to devote greater effort to research and develop new grouting materials, whose price is low, raw material is plentiful, and performance is perfect.

3. Assembled concrete structure is the significant direction in the future. In terms of material, the prospect of grouting material in the vital jointing material of assembled concrete structure is broad. We are supposed to reinforce the research on this area. 


\section{Acknowledgement}

This work is financially supported by the General project of Shenyang Jianzhu university(2015147).

\section{References:}

[1] Hong-Xia Wang, Xing Wang, Yan-Shu He, etc. Improvement and research progress of grouting materials [J]. Concrete, 2008(10):3- 33.

[2] BABAN R, GEYMAYER H. Invcstigations on grouting of resins into joints and cracks of mass concrete [J].Rock and concrete grouting, 1993.

[3] Yuan-Wu, Kai Wang, Xiao-Jing Yang, Shan We. Experimental study on basic mechanical properties of cementitious grout [J].Building Structure. 2014(10):96-99

[4] Shi-Bao Zhang, Ji-Wu Gong, Xi-Xi Lu, etc. Mix proportion experiments research of the grouting material for digging grit holes [J]. Concrete, 2012(2):121-123.

[5] Li-Qiang Huo, Environmental grouting materials with high strength and performace research Concrete, 2013(1):114-116.

[6] Yong-Zhen Wang, Ya-Feng Zhang, Jian-Zheng Guang, etc. Preparation and properties of flexible epoxy grouting material [J].New Chemical Materials, 2011,39(9):88-91.

[7] Xiang-Yang Liu, Xiao-Ming Wu, Hao Liu, etc. Study of new chemical grouting material of a water glass gel [J].Oil Drilling \& Production Technology, 2011,33(5):45-48.

[8] Wen He, Xing-Wang Zhou, Run Xu.Experimental Research on Sodium Silicate Chemical Grouting Material with low Viscosity [J].Coal Science and Technology, 2011,39(4):44-47,77. 